\title{
Reversible Data Hiding Using Hybrid Method of Difference Expansion on Medical Image
}

\author{
Aulia Arham ${ }^{1}$, Ozzy Secio Riza ${ }^{2}$ \\ ${ }^{1,2}$ Department of Information System, Faculty of Science and Technology, \\ State Islamic University Imam Bonjol, Balai Gadang Koto Tangah, Padang 25171, Indonesia
}

\begin{abstract}
Data hiding in the image can cause permanent distortion (irreversible). In other applications such as medical images, misdiagnosis can be caused by distortion in the image. To solve these problems, in the medical image is required a special scheme for embedding data. Reversible data hiding is a scheme that could extract the embedded information and turn in the image to the original image without distortion. Difference Expansion (DE) is the simple scheme in reversible data hiding, where the difference value of two neighboring pixels was used to conceal secret data. In this context, we suggest two schemes based on a hybrid combination of DE; we divide the medical image into two categories: smooth block and non-smooth block areas. The medical image has characteristics that are large in smooth block areas. To evaluate the proposed method, we used four different images. The experimental results showed that embedding capacity and visual quality on both of the proposed schemes are well preserved, respectively evaluated. The proposed scheme has preferable visual quality than the original method, and similar method has been proposed previously. The proposed scheme's embedding capacity is up to $0.66 \mathrm{bpp}$ with the visual quality of PSNR value is up to $48 \mathrm{~dB}$. The proposed method is very suitable for implementation that requires high visual quality, such as in medical images.
\end{abstract}

This work is licensed under a Creative Commons Attribution-Share Alike 4.0

Aulia Arham,

Department of Information System, Faculty of Science and Technology, State Islamic University Imam Bonjol, Balai Gadang Koto Tangah, Padang 25171, Indonesia

Email: auliaarham@uinib.ac.id

\section{INTRODUCTION}

Medical images are the important record of patient's information on the storage media or hospital databases that could be shared via transmission channels between hospitals or health centers and the internet network [1]-[3]. Medical images are commonly obtained from Magnetic Resonance Imaging (MRI), Ultrasound (US), Computerized Radiography, Computed Tomography (CT), and others [4]. Medical image and patient information processing must be controlled precisely to evade misapplication and loss of data. The data storage size must be reduced to enhance the efficiency of bandwidth communication channels.

Data Hiding refers to the embedding of secret data on other media [5]; in other words, the embedding process related to media carriers and secret data embedded [6]-[8]. In many conventional data hiding cases, media carriers can be distorted and cannot be fully restored to the original media after the extraction process. In other implementations, such as medical imagery, military imagery, and law forensics, the media carrier needs to return to the original form after the extraction. Techniques that can handle the case called reversible data hiding, distortion-free, invertible, or lossless techniques. Reversible Data hiding facilitates application possibilities that can restore the media reservoir to their originals after the process of extraction [6], [7], [9][13]. The embedding and extraction process of reversible data hiding is shown in Figure 1 [5].

Reversible data hiding build upon Difference Expansion (DE) is the method that was first introduced by Tian [14]. The difference value of two neighboring pixels was used to conceal secret data, and the average value of the pixel pair was well preserved. This method can embed data with a large capacity. In 2003 and 
2004, [15]-[17] modified the DE method by expanding the number of pixels used in a block Triplets, Quads, and the vector to increase embedding capacity. In 2006, Jaber and Yaqub [18] modified the scheme vector DE [16] by changing the median pixel as the base point used. Hsiao et al. [19] improved the scheme [18] using a block with a size of $3 \times 3$ for the same purpose. In 2012, Peng et al. [20] modified vector DE by combining it with the adaptive embedding. Unlike in previous methods, Liu et al. [21] improved the DE scheme [14] by reducing pixel pairs' expansion value before the embedding process. Hence, the value of the difference after the embedding process is closer to the original value. This scheme is called Reduced Difference Expansion (RDE). Then, in 2009, Yi et al. [22] modified the scheme that was proposed by Liu [21] with the same purpose. This method is called Improved Reduced Difference Expansion (IRDE), where the capacity to embed the data and the visual quality are better than Liu [21]. In 2011, Al-Qershi and Khoo [23] combining some methods of DE that have been proposed previously: the first method made by combining methods from Tian [14] with Chiang et al. [4], the second method from Alattar [17] with Chiang et al. [4]. These methods can embed data with a large capacity in better visual quality. In 2013, Ahmad et al. [24] proposed a new method by combining the methods of Alattar [17] with Liu et al. [21]; this method gives a larger embedding capacity with better visuals. In 2016, Arham et al. [11] proposed a new method for the medical image; the method is the combnation of Chiang et al. [4], Alattar [17], and Yi et al. [22]; this method gives a larger embedding capacity with better visuals in medical images. In 2017, Arham et al. [7] proposed a new method by combining the methods of Alattar [17], and Yi et al. [22]; this method gives a larger embedding capacity with better visuals in multiple layers of data hiding.

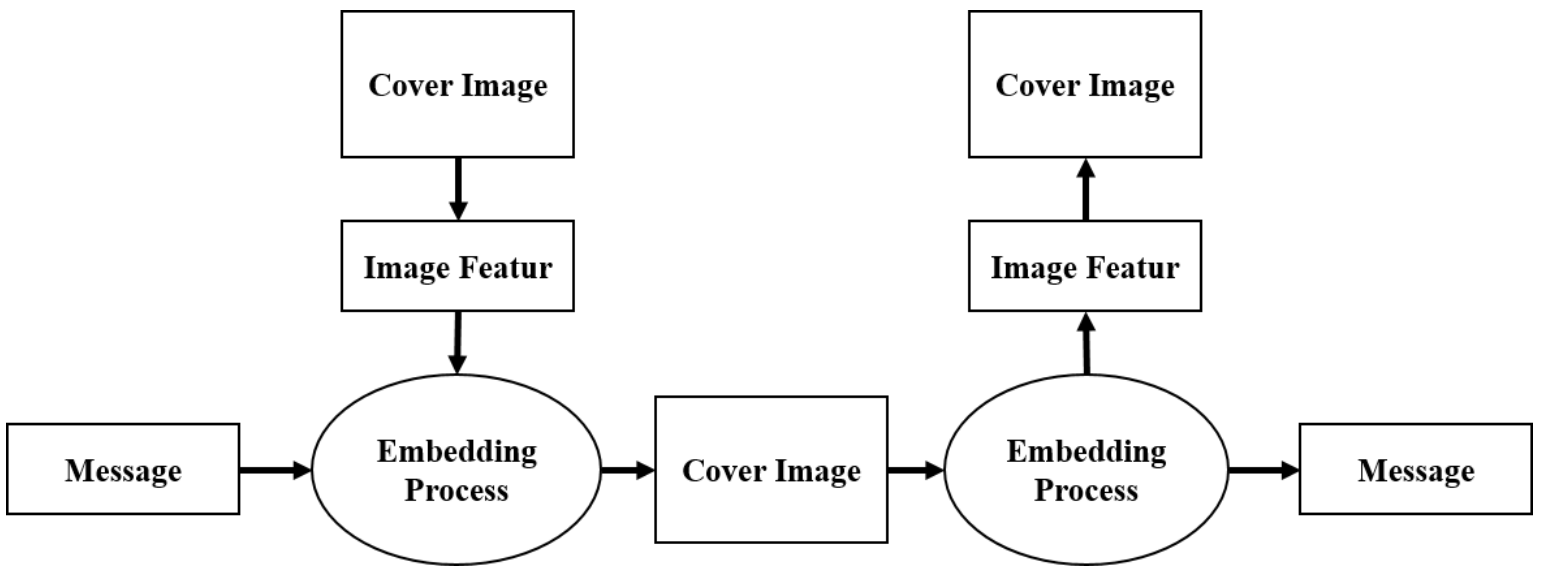

Fig. 1. The procedure of the embedding and extraction process in reversible data hiding.

Previously mentioned schemes have the reversible capability, but similarities (from the original and stegoimage) and the embedding capacity should be improved to use in wider application [20][25]. This study presented a new method that can achieve a large capacity of embedding with better visual quality and suitable medical image applications. It presented two new schemes based on a hybrid combination of DE scheme: the first one is a hybrid combination scheme based on Chiang et al. [4], Tian [14], and Liu et al. [21], and the second one is a hybrid combination based on Chiang et al. [4], Tian [14], and Yi et al. [22]. The hybrid combination schemes of DE aims to take advantage of each scheme based on their characteristics. The Chiang scheme has a high capacity of embedding on the image of smooth and high visual quality. The Tian scheme can be applied to a non-smooth image area with a high embedding capacity but has low visual quality. On the other hand, the Liu and Yi scheme can reduce the expansion value on pixel pairs before the embedding process on the Tian scheme [14], so it can produce good visual quality.

The following sections will be organized as follows. Difference Expansion variations are provided in Section 2. Section 3 describes the proposed schemes. Discussion of the experimental results and comparisons are provided in section 4. Last, the conclusion is given in Section 5.

\section{DIFFERENCE EXPANSION AND ITS VARIATION}

In this section, a brief description will be presented of several various methods of reversible data hiding base on difference expansion that supports the proposed scheme: Difference Expansion (DE), Two-Level DE, Reduced Difference Expansion (RDE), and Improved Reduced Difference Expansion (IRDE). 


\subsection{Difference Expansion (DE)}

DE was first proposed by Tian [14], where this method uses the difference value of a pair of pixels neighboring to the embedding of data while keeping the average value. If $u_{1}$ and $u_{2}$ are the neighbors of the 8bit grayscale image, then the difference value $v$ and average value $m$ of a pair of pixels as it is provided in (1).

$$
\begin{gathered}
m=\frac{u_{1}+u_{2}}{2}, \quad v=u_{1}-u_{2} \\
\tilde{v}=2 \times v+b \\
\tilde{u}_{1}=m+\frac{\tilde{v}+1}{2}, \quad \tilde{u}_{2}=m-\left\lfloor\frac{\tilde{v}}{2}\right\rfloor
\end{gathered}
$$

For the embedding bits data $b$ with the expansion of the difference value pairs of pixels $v$, the embedding process is performed (2). The Difference value pair of pixels $v$ used to create new pixels is provided in (3). In order to avoid overflow and underflow, the value of $\tilde{v}$ must meet the criteria (4).

$$
\left\{\begin{array}{l}
|\tilde{v}| \leq 2 \times(255-m), \text { if } 128 \leq m \leq 255 \\
|\tilde{v}| \leq 2 \times m+1, \text { if } 0 \leq m \leq 127
\end{array}\right.
$$

\subsection{Two-Level DE}

Chiang et al. [4] proposed this method; every $4 \times 4$ pixels can embed 14 bits of data. First, the image is divided into blocks of $4 \times 4$ pixels. The two-level DE transform is then applied to every block. As shown in Figure 2, smooth blocks have all-zero value except for the upper-leftmost pixel after performing the transformation process using Haar wavelet transform.

Non-zero values are embedded in the lower-rightmost pixel, and the upper-leftmost pixel left untouched. Non-zero values are used to identify embedded blocks, and 14 other locations in the block are used to embed the data.

\begin{tabular}{|c|c|c|c|}
\hline $\mathbf{A}$ & $\mathbf{A}$ & $\mathbf{A}$ & $\mathbf{A}$ \\
\hline $\mathbf{A}$ & $\mathbf{A}$ & $\mathbf{A}$ & $\mathbf{A}$ \\
\hline $\mathbf{A}$ & $\mathbf{A}$ & $\mathbf{A}$ & $\mathbf{A}$ \\
\hline $\mathbf{A}$ & $\mathbf{A}$ & $\mathbf{A}$ & $\mathbf{A}$ \\
\hline
\end{tabular}

\begin{tabular}{|l|l|l|l|}
\hline $\mathbf{A}$ & $\mathbf{0}$ & $\mathbf{0}$ & $\mathbf{0}$ \\
\hline $\mathbf{0}$ & $\mathbf{0}$ & $\mathbf{0}$ & $\mathbf{0}$ \\
\hline $\mathbf{0}$ & $\mathbf{0}$ & $\mathbf{0}$ & $\mathbf{0}$ \\
\hline $\mathbf{0}$ & $\mathbf{0}$ & $\mathbf{0}$ & $\mathbf{0}$ \\
\hline
\end{tabular}

Fig. 2. Two-Level DE for Smooth Block [4]

This method is not complicated because it does not need a location map. In addition, the maximal capacity embed is $0.875 \mathrm{bpp}$. This method is appropriate for a particular image that has multiple smooth blocks, such as a medical image.

\subsection{Reduced Difference Expansion (RDE)}

Embedding data with the DE method can cause double the difference value of pixel pairs. From this fact, Liu et al. [21] proposed RDE to reduce the difference value on pixel pairs before data embedding, as it is provided in (5).

$$
\begin{gathered}
\tilde{v}_{1}=\left\{\begin{array}{l}
v_{i}, \text { if } v_{i}<2 \\
v_{i}-2^{\log _{2}|v|-1}, \text { if } v \geq 2
\end{array}\right. \\
L M=\left\{\begin{array}{l}
0, \text { if } 2^{\log _{2}|\tilde{v}|-1}=2^{\log _{2}|v|-1} \text { or } \tilde{v}=v \\
1, \text { if } 2^{\log _{2}|\tilde{v}|-1} \neq 2^{\log _{2}|v|-1}
\end{array}\right. \\
v=\left\{\begin{array}{l}
\tilde{v}+2^{\log _{2}|\tilde{v}|-1}, \text { if } L M=0 \\
\tilde{v}+2^{\log _{2}|\tilde{v}|}, \text { if } L M=1
\end{array}\right.
\end{gathered}
$$

A location map is applied to reconstruct the original difference value whenever this reduction occurs. This location map is specified in (6). The reconstruction process of the original value is performed by using (7). 


\subsection{Improved Reduced Difference Expansion (IRDE)}

This method aims to minimize the difference value of pixel pairs on the DE method before processing for data embedding [21][22]. Yi modifies RDE by minimizing the process performed in (8).

$$
\begin{gathered}
\tilde{v}_{1}=\left\{\begin{array}{l}
v_{i}-2^{\log _{2}|v|-1}, \text { if } 2 \times 2^{n-1} \leq v_{i} \leq 3 \times 2^{n-1}-1 \\
v_{i}-2^{\log _{2}|v|}, \quad \text { if } 3 \times 2^{n-1} \leq v_{i} \leq 4 \times 2^{n-1}-1
\end{array}\right. \\
n=\left\lfloor 2^{\log _{2}|v|-1}\right\rfloor
\end{gathered}
$$

Afterward, the location map is specified in (10), and the reconstruction process of the original value is performed by using (11). From the experimental results by Yi, IRDE has a higher capacity of embedding and better visual quality than RDE.

$$
\begin{gathered}
M= \begin{cases}0, & \text { if } 2 \times 2^{n-1} \leq v_{i} \leq 3 \times 2^{n-1}-1 \\
1, & \text { if } 3 \times 2^{n-1} \leq v_{i} \leq 4 \times 2^{n-1}-1\end{cases} \\
v= \begin{cases}\tilde{v}+2^{\log _{2}|\tilde{v}|+1}, & \text { if } L M=1 \\
\tilde{v}+2^{\log _{2}|\tilde{v}|}, & \text { if } L M=0\end{cases}
\end{gathered}
$$

\section{THE PROPOSED SCHEMES}

The proposed method consists of two schemes based on a hybrid combination of DE on the medical image, where we combine the proposed scheme by Chiang et al. [4], Tian [14], Liu et al. [21], and Yi et al. [22]. The first scheme is a combination of Chiang et al. [4], Tian [14], and Liu et al. [21], and the second scheme is a combination of Chiang et al. [4], Tian [14], and Yi et al. [22]. The objective of combining the three schemes is to take advantage of each scheme based on its characteristics. For a smooth block area of $4 \times 4$ pixels, Tian [14] method could embed maximal 8 bit, whereas Chiang et al. [4] could embed 14 bit. The number of data bits that can be embedded with the Chiang et al. [4] method is zero to the non-smooth block area. It indicates that Chiang et al. [4] scheme is more appropriate to a smooth block area. Then, we propose a new scheme where the Chiang method is used for embedding the smooth block area and Tian [14] for embedding the non-smooth block area. The method of Liu et al. [21] and Yi et al. [22] are used to reduce the value differences of pixel pairs in Tian [14] scheme to get a better visual image after embedding data.

\subsection{Embedding Process}

The embedding process consists of the following steps:

1. Divide the original image into a non-overlapping block of $4 \times 4$ pixels.

2. Check blocks sequentially and classified into two types: smooth block or non-smooth area.

3. Use the non-smooth block area and then reducing the value difference between pixel pairs with the method by [21] or [22], and embedding process using [14]. Embedded Map formed is then compressed. If the block's number of non-smooth is sufficient to hide all the data bits, then continue to step 5.

4. Concatenate the rest of the embedded data with the compressed embedding map.

5. Embed data from step 3 or 4 into the smooth block area with Chiang et al. [4]'s method.

\subsection{Extraction Process}

The extraction process goals are to gain the hidden secret message with the step as follows:

1. Divide the original image into a non-overlapping block of $4 \times 4$.

2. Check blocks sequentially. Blocks used during embedding should be identified with zero value and, if found, categorized the block into the smooth block. Extract 14 bits, and the value of the original pixels are redeemed.

3. Embedding Map compressed gain from the prior move, and then decompressed, also obtained the second part from the residue of the embedded data.

4. Use an embedding map to extract data using Tian [14] and restore the original value of pixels using a method by Liu et al. [21] or Yi et al. [22].

\section{EXPERIMENTAL RESULTS AND COMPARISON}

The data used to evaluate the proposed method performance are random from six embedding schemes: from 0.1 to $0.6 \mathrm{bpp}$. Then, the results are compared with methods from previous researchers. There are four gray-scale medical images used, as shown in Figure 3. 
The Peak-Signal-to-Noise-Ratio (PSNR) quads as described in equation (26), and the Structural Similarity Index Measure (SSIM) quads as described in equation (28) were used to evaluate the similarity of stego-image with an original image. The degree of similarity is better indicated by a high PSNR value, but instead, a better degree of similarity indicated by a small MSE value. SSIM is a method for measuring difference between two similar images. The SSIM index is a perceptual metric that quantifies image quality degradation; in other words, image quality is measured or predicted based on a distortion-free or uncompression initial image as a reference. The SSIM index used value between -1 and 1 , where the value one gain when a pair of images are identical.

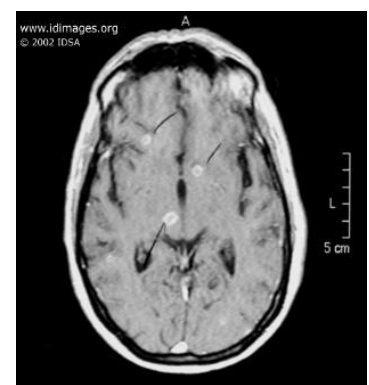

MR

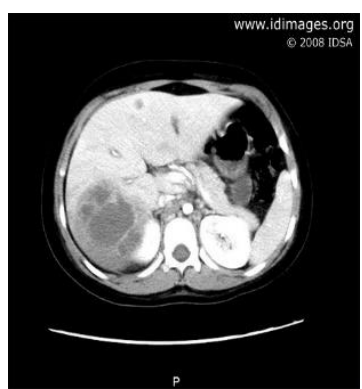

CT

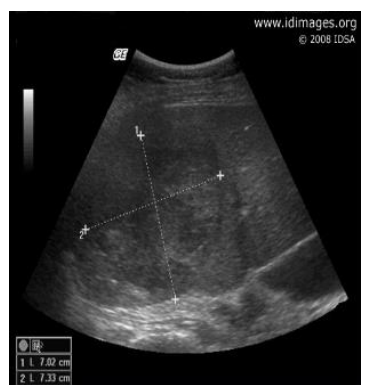

US

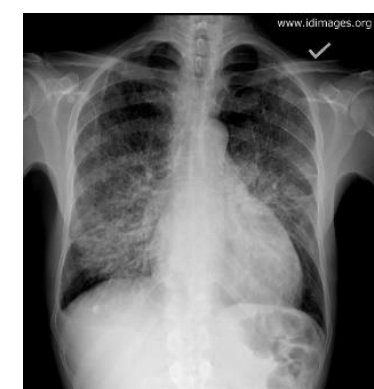

X-Ray

Fig. 3. Eight medical images that are used to evaluate [27]

$$
\begin{gathered}
\operatorname{PSNR}\left(I, I_{W}\right)=10 \log _{10} \frac{255^{2}}{\operatorname{MSE}\left(I, I_{W}\right)} \\
\operatorname{MSE}\left(I, I_{W}\right)=\frac{1}{m \times n} \sum_{i}^{m-1} \sum_{j}^{n-1}\left|I(i, j)-I_{W}(i, j)\right|^{2} \\
\operatorname{SSIM}\left(I, I_{W}\right)=\frac{\left(2_{\mu_{I} \mu_{I}}+c_{1}\right)\left(2 \operatorname{cov}+c_{2}\right)}{\left(\mu_{I}^{2}+\mu_{I_{W}}^{2}+c_{1}\right)\left(\sigma_{I}^{2}+\sigma_{I_{W}}^{2}+c_{2}\right)}
\end{gathered}
$$

When $I$ is original images, $I_{W}$ is stego-images, $\mu_{I}$ and $\mu_{I_{W}}$ is the average of $I$ and $I_{W}, \sigma_{I}^{2}$ and $\sigma_{I_{W}}^{2}$ is a variant of $I$ and $I_{W}$.

The results showed that the proposed method has high embedding capacity. As seen in Table 1 that the proposed method has the embedding capacity up to $0.66 \mathrm{bpp}$. The embedding capacity of [11] had the highest value compared to other method for all images used. The Comparison of embedding capacity for two schemes proposed with five other schemes can be seen in Table 2 and Table 3. The two proposed schemes have increased in CT and US images compared to Ahmad [24] and Arham [7], and in MR image compare to Al-Qershi \& Khoo [23] P1. The improvement of the first proposed method in CT image is 0.2834 bpp compared to Ahmad [24] and 0.2525 bpp compared to Arham [7], In US image has an improvement of 0.1320 bpp compared to Ahmad [24] and 0.1232 bpp compared to Arham [7], and In MR image has an improvement of $0.0136 \mathrm{bpp}$ compared to Al-Qershi \& Khoo [23] P1.

The improvement of the second proposed method in CT image is 0.2868 bpp compared to Ahmad [24] and $0.2555 \mathrm{bpp}$ compared to Arham [7], In US image has an improvement of $0.1354 \mathrm{bpp}$ compared to Ahmad [24] and 0.1266 bpp compared to Arham [7], and In MR image has an improvement of 0.0170 bpp compared to Al-Qershi \& Khoo [23] P1. Compared to the Al-Qershi \& Khoo [23] P2 and Arham scheme [11] the proposed scheme always decreases for all images used. The deterioration of the first proposed method compared to Al-Qershi \& Khoo [23] P2 has the average decrease is $0.1572 \mathrm{bpp}$ and $0.1985 \mathrm{bpp}$ compared to Arham [11]. The deterioration of the second proposed method compared to Al-Qershi \& Khoo [23] P2 has the average decrease is $0.1538 \mathrm{bpp}$ and $0.1538 \mathrm{bpp}$ compared to Arham [11].

A Comparison of visual quality for two schemes proposed by a number of other schemes can be seen in Figure 4 and Table 4. In Figure 4 shown, the proposed method has better visual quality from other schemes as indicated by the high value of PSNR for all images used. In Figure 4(a) we can see, with the embedding of 0.5 bpp the proposed method has a higher value of PSNR, where the first proposed method has $44.89 \mathrm{db}$ and the second proposed method has $46.91 \mathrm{db}$. In Figure 4(b) with the embedding of $0.6 \mathrm{bpp}$, proposed method has a higher value of PSNR, where the first proposed method has $46.03 \mathrm{db}$ and the second proposed method has 
$48.07 \mathrm{db}$. In Figure 4(c) with the embedding of $0.6 \mathrm{bpp}$, proposed method has a higher value of PSNR, where the first proposed method has $48.54 \mathrm{db}$ and the second proposed method has $50.27 \mathrm{db}$. In Figure 4(d) with the embedding of $0.5 \mathrm{bpp}$, proposed method has a higher value of PSNR, where the first proposed method has $48.00 \mathrm{db}$ and the second proposed method has $50.52 \mathrm{db}$. In Table 4, the proposed method had a high value of SSIM, where the SSIM value that was always higher than 0.980 for each maximum embedded in all images used.

Table 1. Comparison between the embedding capacities

\begin{tabular}{lcccc}
\hline \multirow{2}{*}{ Schemes } & \multicolumn{4}{c}{ Available Embedding Capacity (bpp) } \\
\cline { 2 - 5 } & MR & CT & US & X-Ray \\
\hline Al-Qershi \& Khoo [23] P1 & 0.5196 & 0.6312 & 0.6217 & 0.5448 \\
Al-Qershi \& Khoo [23] P2 & 0.7033 & 0.6083 & 0.7118 & 0.7384 \\
Ahmad [24] & 0.6335 & 0.2498 & 0.4012 & 0.6241 \\
Arham [7] & 0.6401 & 0.2811 & 0.4100 & 0.6274 \\
Arham [11] & 0.7427 & 0.6957 & 0.7419 & 0.7468 \\
Proposed Scheme 1 & 0.5332 & 0.6536 & 0.6293 & 0.5466 \\
Proposed Scheme 2 & 0.5366 & 0.6639 & 0.6316 & 0.5471 \\
\hline
\end{tabular}

Table 2. Average increase in the embedding capacity of first proposed scheme 1

\begin{tabular}{lrrrrr}
\hline \multirow{2}{*}{ Schemes } & \multicolumn{7}{c}{ Proposed Scheme 1 } & & \\
\cline { 2 - 6 } & MR & CT & US & X-Ray & Average \\
\hline Al-Qershi \& Khoo [23] P1 & 0.0136 & -0.0979 & -0.0885 & -0.0115 & -0.0461 \\
Al-Qershi \& Khoo [23] P2 & -0.1700 & -0.0750 & -0.1786 & -0.2052 & -0.1572 \\
Ahmad [24] & -0.1003 & 0.2834 & 0.1320 & -0.0908 & 0.0561 \\
Arham [7] & -0.1068 & 0.2522 & 0.1232 & -0.0942 & 0.0436 \\
Arham [11] & -0.2095 & -0.1624 & -0.2087 & -0.2135 & -0.1985 \\
\hline
\end{tabular}

Table 3. Average increase in the embedding capacity of second proposed scheme 2

\begin{tabular}{lccccc}
\hline \multirow{2}{*}{ Schemes } & \multicolumn{7}{l}{ Proposed Scheme 2 } & & \\
\cline { 2 - 6 } & MR & CT & US & X-Ray & \multicolumn{1}{c}{ Average } \\
\hline Al-Qershi \& Khoo [23] P1 & 0.0170 & -0.0946 & -0.0851 & -0.0082 & -0.0427 \\
Al-Qershi \& Khoo [23] P2 & -0.1667 & -0.0717 & -0.1752 & -0.2018 & -0.1538 \\
Ahmad [24] & -0.0969 & 0.2868 & 0.1354 & -0.0875 & 0.0594 \\
Arham [7] & -0.1035 & 0.2555 & 0.1266 & -0.0908 & 0.0470 \\
Arham [11] & -0.2061 & -0.1591 & -0.2053 & -0.2102 & -0.1952 \\
\hline
\end{tabular}

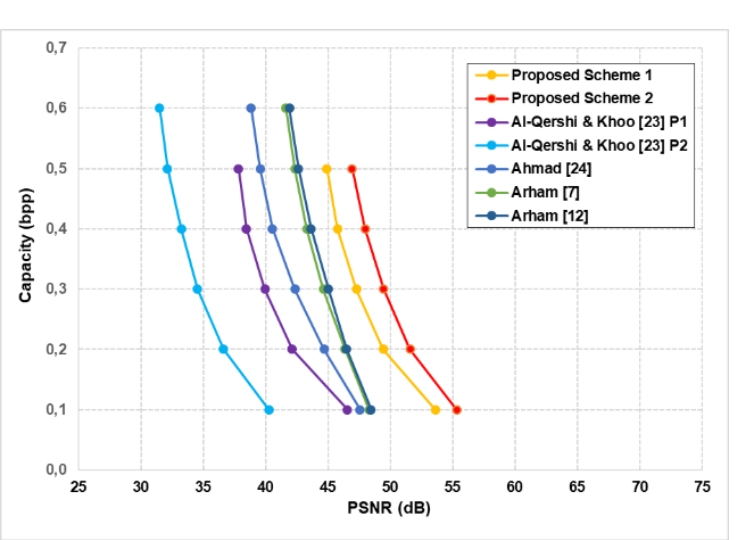

(a)

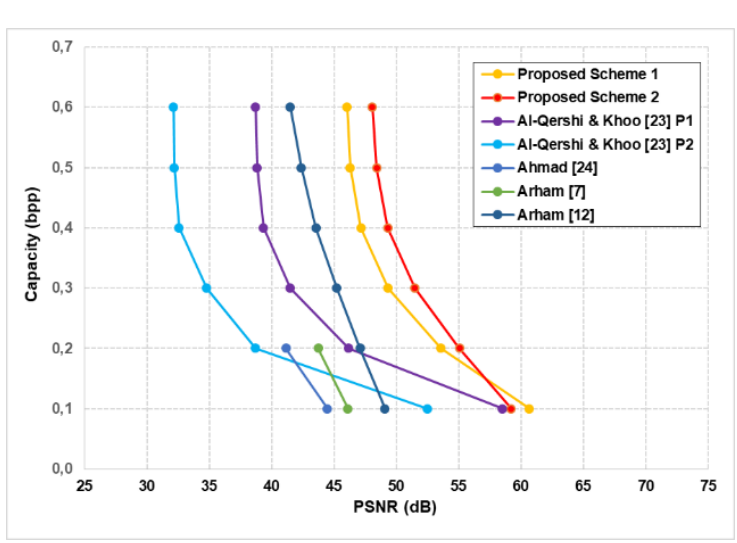

(b) 


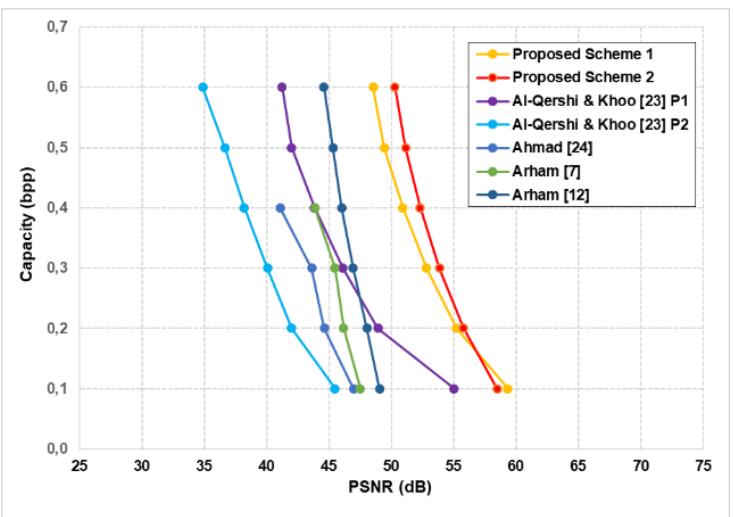

(c)

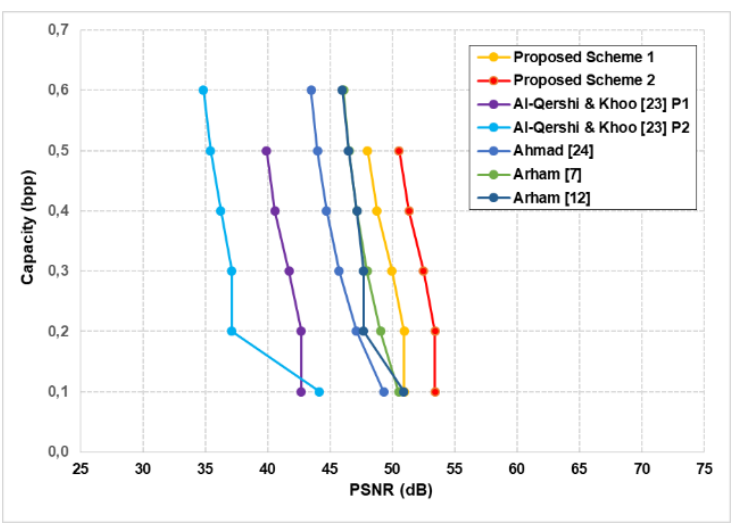

(d)

Fig. 4. A comparison of the actual embedding capacities and visual quality. (a) MR. (b) CT. (c) US. (d) XRay

Table 4. comparison between the proposed schemes with 5 schemes in terms of SSIM

\begin{tabular}{|c|c|c|c|c|c|c|c|}
\hline \multirow{2}{*}{ Image } & \multirow{2}{*}{ Schemes } & \multicolumn{6}{|c|}{ SSIM } \\
\hline & & $0.1 \mathrm{bpp}$ & $0.2 \mathrm{bpp}$ & $0.3 \mathrm{bpp}$ & $0.4 \mathrm{bpp}$ & $0.5 \mathrm{bpp}$ & $0.6 \mathrm{bpp}$ \\
\hline \multirow{7}{*}{ MR } & Al-Qershi \& Khoo [23] P1 & 0.997 & 0.990 & 0.984 & 0.978 & 0.974 & \\
\hline & Al-Qershi \& Khoo [23] P2 & 0.993 & 0.982 & 0.970 & 0.957 & 0.945 & 0.933 \\
\hline & Ahmad [24] & 0.998 & 0.996 & 0.993 & 0.991 & 0.988 & 0.986 \\
\hline & Arham [7] & 0.998 & 0.997 & 0.995 & 0.994 & 0.992 & 0.991 \\
\hline & Arham [11] & 0.998 & 0.996 & 0.993 & 0.992 & 0.990 & 0.988 \\
\hline & Proposed Scheme 1 & 0.998 & 0.996 & 0.994 & 0.993 & 0.991 & \\
\hline & Proposed Scheme 2 & 0.998 & 0.997 & 0.995 & 0.994 & 0.992 & \\
\hline \multirow{7}{*}{$\mathrm{CT}$} & Al-Qershi \& Khoo [23] P1 & 0.997 & 0.991 & 0.984 & 0.978 & 0.973 & 0.970 \\
\hline & Al-Qershi \& Khoo [23] P2 & 0.996 & 0.985 & 0.968 & 0.953 & 0.947 & 0.944 \\
\hline & Ahmad [24] & 0.997 & 0.994 & & & & \\
\hline & Arham [7] & 0.999 & 0.997 & & & & \\
\hline & Arham [11] & 0.997 & 0.994 & 0.992 & 0.990 & 0.987 & 0.984 \\
\hline & Proposed Scheme 1 & 0.997 & 0.994 & 0.991 & 0.989 & 0.987 & 0.983 \\
\hline & Proposed Scheme 2 & 0.997 & 0.994 & 0.992 & 0.990 & 0.988 & 0.985 \\
\hline \multirow{7}{*}{ US } & Al-Qershi \& Khoo [23] P1 & 0.997 & 0.992 & 0.985 & 0.979 & 0.972 & 0.969 \\
\hline & Al-Qershi \& Khoo [23] P2 & 0.995 & 0.985 & 0.973 & 0.959 & 0.943 & 0.927 \\
\hline & Ahmad [24] & 0.997 & 0.994 & 0.992 & 0.990 & & \\
\hline & Arham [7] & 0.999 & 0.997 & 0.996 & 0.995 & & \\
\hline & Arham [11] & 0.997 & 0.994 & 0.991 & 0.989 & 0.987 & 0.986 \\
\hline & Proposed Scheme 1 & 0.997 & 0.994 & 0.991 & 0.989 & 0.987 & 0.985 \\
\hline & Proposed Scheme 2 & 0.997 & 0.994 & 0.992 & 0.990 & 0.988 & 0.986 \\
\hline \multirow{7}{*}{ X-Ray } & Al-Qershi \& Khoo [23] P1 & 0.980 & 0.980 & 0.974 & 0.966 & 0.958 & \\
\hline & Al-Qershi \& Khoo [23] P2 & 0.990 & 0.946 & 0.946 & 0.933 & 0.917 & 0.903 \\
\hline & Ahmad [24] & 0.998 & 0.995 & 0.992 & 0.989 & 0.986 & 0.984 \\
\hline & Arham [7] & 0.999 & 0.997 & 0.996 & 0.994 & 0.993 & 0.991 \\
\hline & Arham [11] & 0.998 & 0.994 & 0.994 & 0.993 & 0.991 & 0.989 \\
\hline & Proposed Scheme 1 & 0.995 & 0.995 & 0.994 & 0.992 & 0.990 & \\
\hline & Proposed Scheme 2 & 0.997 & 0.997 & 0.996 & 0.994 & 0.992 & \\
\hline
\end{tabular}

From the experimental results obtained, by performing reduction of the method by Tian [14] may enhance the image's embedding capacity and quality visual. Between the two proposed method, the second method has a higher capacity and better visual quality, the embedding capacity reaches the highest ratio in the CT image with an increase of $0.0103 \mathrm{bpp}$, and better visual quality indicated by the highest value of PSNR and SSIM. 


\section{CONCLUSION}

In recent years, many researchers have been proposed methods to embed data in other media. However, some methods may not work well on the medical image. Large embedding capacity is not the main goal in implementing reversible data hiding in a medical image; however, the visual quality of the image after the embedding process needs to be considered.

This study presents two methods based on a hybrid combination of DE on the medical image. In both proposed methods, the embedding process is similar to Al-Qershi \& Khoo method [23]. However, we reduce the expansion value on pixel pairs before the embedding process on Tian [14] with the method as that of Yi et al. [22] or Liu et al. Both proposed methods are simple with low complexity. Proposed methods have a high embedding capacity and better visual quality than original methods and similar methods that have been previously proposed based on the experimental results shown. The proposed method's visual quality has improved in the non-smooth block area after the embedding process indicated by the increase in PSNR and SSIM. The proposed scheme is very suitable for applications that require high visual quality, such as in medical images.

\section{REFERENCES}

[1] V. M. Manikandan, N. L. Prasad, and M. Vedhanayagam, "Half Difference Expansion Based Reversible Data Hiding Scheme for Medical Image Forensics," Curr. Med. Imaging, vol. 16, no. 4, pp. 383-396, 2020. DOI: https://doi.org/10.2174/1573405614666180903120018

[2] M. Fadhil et al., "Secure Reversible Data Hiding in the Medical Image using Histogram Shifting and RC4 Encryption," in 2019 International Seminar on Application for Technology of Information and Communication (iSemantic), 2019, pp. 1-6.

[3] Y. Yang, W. Zhang, D. Liang, and N. Yu, "A ROI-based high capacity reversible data hiding scheme with contrast enhancement for medical images," Multimed. Tools Appl., vol. 77, no. 14, pp. 18043-18065, 2018. DOI: https://doi.org/10.1007/s11042-017-4444-0

[4] K.-H. Chiang, K.-C. Chang-Chien, R.-F. Chang, and H.-Y. Yen, "Tamper detection and restoring system for medical images using wavelet-based reversible data embedding," J. Digit. Imaging, vol. 21, no. 1, pp. 77-90, 2008. DOI: https://doi.org/10.1007/s10278-007-9012-0

[5] T.-C. Lu and T. N. Vo, "Introduction of Reversible Data Hiding Schemes," in International Conference on Security with Intelligent Computing and Big-data Services, 2019, pp. 170-183. DOI: https://doi.org/10.1007/978-3-03046828-6_15

[6] Z. Ni, Y.-Q. Shi, N. Ansari, and W. Su, "Reversible data hiding," IEEE Trans. Circuits Syst. Video Technol., vol. 16, no. 3, pp. 354-362, 2006. DOI: https://doi.org/10.1109/TCSVT.2006.869964

[7] A. Arham, H. A. Nugroho, and T. B. Adji, "Multiple Layer Data Hiding Scheme Based on Difference Expansion of Quad," Signal Processing, vol. 137, pp. 52-62, 2017, doi: 10.1016/j.sigpro.2017.02.001. DOI: https://doi.org/10.1016/j.sigpro.2017.02.001

[8] P. Maniriho and T. Ahmad, "Information hiding scheme for digital images using difference expansion and modulus function," J. King Saud Univ. Inf. Sci., vol. 31, no. 3, pp. 335-347, 2019. DOI: https://doi.org/10.1016/j.jksuci.2018.01.011

[9] O. M. Al-Qershi and B. E. Khoo, "Two-dimensional difference expansion (2D-DE) scheme with a characteristicsbased threshold," Signal Processing, vol. 93, no. 1, pp. 154-162, 2013. DOI: https://doi.org/10.1016/j.sigpro.2012.07.012

[10] T.-S. Nguyen and C.-C. Chang, "A Reversible Data Hiding Scheme Based on the Sudoku Technique," Displays, vol. 39, pp. 109-116, Oct. 2015, DOI: https://doi.org/10.1016/j.displa.2015.10.003

[11] A. Arham, H. A. Nugroho, and T. B. Adji, "Combination schemes Reversible Data Hiding for medical images," in Science and Technology-Computer (ICST), International Conference on, 2016, pp. 44-49. DOI: https://doi.org/10.1109/ICSTC.2016.7877345

[12] P. Maniriho and T. Ahmad, "Enhancing the Capability of Data Hiding Method Based on Reduced Difference Expansion.," Eng. Lett., vol. 26, no. 1, 2018.

[13] C.-F. Lee, J. J. Shen, and Y. H. Lai, "Data hiding using multi-pixel difference expansion," in 2018 3rd International Conference on Computer and Communication Systems (ICCCS), 2018, pp. 56-60. DOI: https://doi.org/10.1109/CCOMS.2018.8463244

[14] J. Tian, "Reversible data embedding using a difference expansion," IEEE Trans. Circuits Syst. Video Technol., vol. 13, no. 8, pp. 890-896, 2003. DOI: https://doi.org/10.1109/TCSVT.2003.815962

[15] A. M. Alattar, "Reversible watermark using difference expansion of triplets," in IEEE International Conference on Image Processing, ICIP'2003', 2003, vol. 1, pp. I-501-4 vol.1, doi: https://doi.org/10.1109/ICIP.2003.1247008

[16] A. M. Alattar, "Reversible watermark using difference expansion of quads," in IEEE International Conference on Acoustics, Speech, and Signal Processing, 2004, vol. 3, pp. iii-377-80 vol.3, DOI: 10.1109/ICASSP.2004.1326560

[17] A. M. Alattar, "Reversible watermark using the difference expansion of a generalized integer transform," IEEE Trans. Image Process., vol. 13, no. 8, pp. 1147-1156, 2004, DOI: https://doi.org/10.1109/TIP.2004.828418

[18] A. Al-Jaber and M. K. Yaqub, "Reversible Watermarking Using Modified Difference Expansion," Int. J. Comput. Inf. Sci., vol. 4, no. 3, p. 134, 2006.

[19] J.-Y. Hsiao, K.-F. Chan, and J. M. Chang, "Block-based reversible data embedding," Signal Processing, vol. 89, no. 
4, pp. 556-569, 2009. DOI: https://doi.org/10.1016/j.sigpro.2008.10.018

[20] F. Peng, X. Li, and B. Yang, "Adaptive reversible data hiding scheme based on integer transform," Signal Processing, vol. 92, no. 1, pp. 54-62, Jan. 2012, DOI: https://doi.org/10.1016/j.sigpro.2011.06.006

[21] C.-L. Liu, D.-C. Lou, and C.-C. Lee, "Reversible Data Embedding Using Reduced Difference Expansion," in Third International Conference on Intelligent Information Hiding and Multimedia Signal Processing, 2007, vol. 1, pp. 433436, DOI: https://doi.org/10.1109/IIH-MSP.2007.267.

[22] H. Yi, S. Wei, and H. Jianjun, "Improved reduced difference expansion based reversible data hiding scheme for digital images," in International Conference on Electronic Measurement \& Instruments, 2009, pp. 4-318, DOI: https://doi.org/10.1109/ICEMI.2009.5274054

[23] O. M. Al-Qershi and B. E. Khoo, "High capacity data hiding schemes for medical images based on difference expansion," J. Syst. Softw., vol. 84, no. 1, pp. 105-112, 2011. DOI: https://doi.org/10.1016/j.jss.2010.08.055

[24] T. Ahmad, M. Holil, and W. Wibisono, "An improved Quad and RDE-based medical data hiding method," in IEEE International Conference on Computational Intelligence and Cybernetics, 2013, pp. 141-145.

[25] M. Tang, J. Hu, and W. Song, "A high capacity image steganography using multi-layer embedding," Opt. - Int. J. Light Electron Opt., vol. 125, no. 15, pp. 3972-3976, Aug. 2014, DOI: https://doi.org/10.1016/j.ijleo.2014.01.149

[26] Z. Wang, A. C. Bovik, H. R. Sheikh, and E. P. Simoncelli, "Image quality assessment: from error visibility to structural similarity," IEEE Trans. Image Process., vol. 13, no. 4, pp. 600-612, 2004, DOI: https://doi.org/10.1109/TIP.2003.819861

[27] "(2016, Jun) Partners Infectious Disesase Images, Emicrobes Digital Library." http://www.idimages.org/images/browse/ImageTechnique/.

\section{BIOGRAPHY OF AUTHORS}

Aulia Arham was born in Solok, Sumatera Barat, Indonesia on July 18, 1990. He received the B.Sc. degrees in Informatics Engineering from the Sekolah Tinggi Manajemen Informatika dan Komputer Duta Bangsa (STMIKDB), Surakarta, Indonesia and the M.S. degree in Electrical Engineering atUniversitas Gadjah Mada, Yogyakarta, Indonesia, in 2016. His research interests are Watermarking, Steganography, Cryptography, Security, Image Processing and Medical Imaging.

Ozzy Secio Riza was born in Padang, Sumatera Barat, Indonesia on October 12, 1993. She received the master degree in Informatics at Sepuluh Nopember Institute of Technology, Surabaya, Indonesia, in 2018. Her research interests are Image Processing, Medical Imaging, and Computational Intelligence. 